TRANSACTIONS OF THE

AMERICAN MATHEMATICAL SOCIETY

Volume 355, Number 4, Pages 1579-1591

S 0002-9947(02)03215-4

Article electronically published on November 18, 2002

\title{
APPROXIMATION OF PLURISUBHARMONIC FUNCTIONS BY MULTIPOLE GREEN FUNCTIONS
}

\author{
EVGENY A. POLETSKY
}

\begin{abstract}
For a strongly hyperconvex domain $D \subset \mathbb{C}^{n}$ we prove that multipole pluricomplex Green functions are dense in the cone in $L^{1}(D)$ of negative plurisubharmonic functions with zero boundary values.
\end{abstract}

\section{INTRODUCTION}

Pluripotential theory for studying plurisubharmonic functions is more important for complex analysis in several variables than potential theory for complex analysis in one variable because the theory in several variables lacks the multitude of methods that can be used in the classical case.

However, many beautiful results of potential theory are still either unproven or wrong in pluripotential theory. For example, the Riesz Representation Theorem states, firstly, that a subharmonic function on a nice domain $D$ is a sum of a subharmonic function with zero boundary values and a harmonic function. Secondly, a subharmonic function $u$ with zero boundary values is the limit of linear combinations with positive coefficients of Green functions in $L^{1}(D)$. So this important theorem gives a complete description of such functions.

The first statement does not hold for plurisubharmonic functions if harmonic functions are replaced by pluriharmonic. The second one immediately meets several obstacles. First of all, in potential theory a linear combination with positive coefficients of Green functions is harmonic outside of its poles. In pluripotential theory there is an analog of Green functions introduced by V. P. Zahariuta in [Z] and M. Klimek in [K1] and called pluricomplex Green functions. They are maximal, i.e., $\left(d d^{c} g\right)^{n}=0$ outside of the poles, but for a sum $u$ of pluricomplex Green functions $\left(d d^{c} u\right)^{n}$, in general, is not zero outside the poles. (The operator $\left(d d^{c} u\right)^{n}$ is an analog of the Laplace operator for plurisubharmonic functions.)

Although the absence of linear functional analysis does not allow us to expand the old methods to the new situation, in this paper we prove that multipole pluricomplex Green functions (see \$2) are dense in the cone in $L^{1}(D)$ of negative plurisubharmonic functions with zero boundary values. Doing this, we establish the second part of the Riesz Representation Theorem.

A particular case of this problem is the approximation of the relative extremal function $\omega$ of a pluriregular compact set $K$ (see \$2). It was shown by V. P. Zahariuta

Received by the editors August 28, 2001.

2000 Mathematics Subject Classification. Primary 32U35; Secondary 32U15.

Key words and phrases. Pluricomplex Green functions.

The author was partially supported by NSF Grant DMS-9804755.

(C)2002 American Mathematical Society 
in [Z] that the existence of approximations with uniform convergence outside $K$ implies a Kolmogorov conjecture regarding entropies of compact sets in $\mathbb{C}^{n}$ (see also [Z1]). In [ZS] V. P. Zahariuta and N. P. Skiba confirmed the existence of approximations when $n=1$. The existence of such approximations in several variables was conjectured by Zahariuta (see [Z] and [Z1]) but stayed unproven for many years. Recently A. Aytuna, A. Rashkovskii and V. P. Zahariuta proved it for pairs of Reinhardt domains (see [ARZ]).

To achieve our goal we start in 3 with relative extremal functions $\omega$ of multiple condensers. It was known from the famous paper of E. Bishop $[\mathrm{B}]$ that it is possible to approximate compact sets in $\mathbb{C}^{n}$ by analytic polyhedra defined by $n$ holomorphic functions $f_{1 j}, \ldots, f_{n j}$. Since $g_{j}=\log \max \left\{\left|f_{1 j}\right|, \ldots,\left|f_{n j}\right|\right\}$ has logarithmic poles and is maximal outside of them, they are almost multipole Green functions, except for having zero values at the boundary.

It was shown by S. Nivoche in $[\mathrm{N}]$ that in the case of a simple condenser Bishop's construction also approximates normal derivatives of $\omega$ by normal derivatives of $g_{j}$ near $K$. In 93 we do it for a multiple condenser.

In $\$ 4$ we show that Stokes' theorem in the form of the Comparison Principle implies that the Monge-Ampère masses are also approximated. For a simple condenser this result was obtained in $[\mathrm{N}]$. It was shown in [NP] that to prove Zahariuta's conjecture it suffices to construct multipole Green functions $g_{j}$ with poles near $K$ that are greater than $\omega$ near $K$ but the total Monge-Ampère mass of $g_{j}$ converges to the total Monge-Ampère mass of $\omega$. The usage of this method in [N] led to the proof of Zahariuta's and, consequently, Kolmogorov's conjecture. We use a similar approach to prove the existence of an approximation of $\omega$ by multipole Green functions.

After that the proof of the main result in $₫ 5$ follows from the possibility of approximating any continuous plurisubharmonic function uniformly by relative extremal functions of condensers and a general plurisubharmonic function by continuous plurisubharmonic functions.

\section{Preliminary Results}

An open set $D \subset \mathbb{C}^{n}$ is strongly hyperconvex if there is a continuous plurisubharmonic function $\phi$ on a neighborhood $V$ of $\bar{D}$ and $D=\{\phi<0\}$. The function $\phi$ is called an exhaustion function.

A negative function $u$ on $D$ has zero boundary values if

$$
\liminf _{z \rightarrow \partial D} u(z)=0 .
$$

Suppose that $W=\left\{w_{1}, \ldots, w_{m}\right\}$ is a finite set in $D$. We say that a plurisubharmonic function $u$ is maximal outside $W$ and has logarithmic poles at points of $W$ if for every $w_{j} \in W$ there are a number $a_{j}>0$, called the weight of $u$ at $w_{j}$, and a number $c$ such that

$$
a_{j} \log \left|z-w_{j}\right|-c \leq u(z) \leq a_{j} \log \left|z-w_{j}\right|+c
$$

near $w_{j}, u$ is locally bounded on $D \backslash W$ and $\left(d d^{c} u\right)^{n}=0$ on $D \backslash W$.

For such functions the Monge-Ampère operator can still be reasonably defined. For example, the Comparison Principle still holds (see [K2 Ch. 6]). Also (see [K2] 
or $[\mathrm{D}]$ )

$$
\left(d d^{c} u\right)^{n}=(2 \pi)^{n} \sum_{j=1}^{m} a_{j}^{n} \delta_{w_{j}}
$$

and if boundary values of $u$ are strictly greater than $c$, then

$$
\int_{D}\left(d d^{c} \max \{u, c\}\right)^{n}=(2 \pi)^{n} \sum_{j=1}^{m} a_{j}^{n} .
$$

As an example of such a function, one can take any holomorphic functions $f_{1}, \ldots, f_{n}$ on $D$ such that the system $f_{1}=\cdots=f_{n}=0$ has simple zeros at points of $W$. Then the function $v=\log \max \left\{\left|f_{1}\right|, \ldots,\left|f_{n}\right|\right\}$ is maximal outside $W$ and has logarithmic poles of weight 1 at every point of $W$.

If $D$ is strongly hyperconvex, then for every choice of weights $a_{j}$ there is a plurisubharmonic function $g_{D}(z, W)$ that is continuous and maximal outside $W$, has logarithmic poles of weight $a_{j}$ at points of $W$ and has zero boundary values. This function is called a multipole pluricomplex Green function. We will always call it just the Green function.

A pluriregular condenser $K=\left(K_{1}, \ldots, K_{m}, \sigma_{1}, \ldots, \sigma_{m}\right)$ is a system of pluriregular compact sets

$$
K_{m} \subset K_{m-1} \subset \cdots \subset K_{1} \subset D \subset \bar{D}=K_{0}
$$

and numbers $\sigma_{m}<\sigma_{m-1}<\cdots<\sigma_{1}<\sigma_{0}=0$ such that there is a continuous plurisubharmonic function $\omega(z)=\omega(z, K, D)$ on $D$ with zero boundary values, $K_{i}=\left\{\omega \leq \sigma_{i}\right\}$ and $\omega$ is maximal on the complement of $K_{i}$ in the interior of $K_{i-1}$ for all $1 \leq i \leq m$. We will call this function the relative extremal function of the condenser $K$ in $D$. Of course, not every choice of sets $K_{i}$ and numbers $\sigma_{i}$ can be realized as a condenser. But if $u$ is a continuous negative plurisubharmonic function on $D$ and the sets $K_{i}=\left\{u \leq \sigma_{i}\right\}$ are pluriregular, then $K$ has a continuous relative extremal function.

The ball of radius $r$ centered at $z$ will be denoted by $B(z, r)$. Also, $S(z, r)=$ $\partial B(z, r)$, and $m(A)$ is the Lebesgue measure of $A$.

\section{Approximation of CONDENSERS By holomorphic FunCtions}

This section further develops the approach of $[\mathbb{N}$.

Let $K=\left(K_{1}, \ldots, K_{m}, \sigma_{1}, \ldots, \sigma_{m}\right)$ be a pluriregular condenser in a strongly hyperconvex open set $D$ with an exhaustion function $\phi$ defined on a neighborhood $V$ of $\bar{D}$. We assume that the sets $D^{j}=\{\phi<1 / j\}$ compactly belong to $V$ for all $j=1,2, \ldots$ Let $\omega(z)=\omega(z, K, D)$ and $D_{r}=\{z \in D: \omega(z)<r\}$.

Suppose that $f_{1}, \ldots, f_{N}$ are holomorphic functions on some $D^{j}, p$ is a positive integer and

$$
v(z)=\sup _{1 \leq k \leq N} \frac{1}{p} \log \left|f_{k}(z)\right| .
$$

We say that the functions $f_{1}, \ldots, f_{N}$ and the integer $p$ approximate $K$ for $\epsilon>0$ if for all $1 \leq i \leq m$ there are numbers $\epsilon_{i}, 0<\epsilon_{i} \leq \epsilon$, such that:

(1) $\sigma_{i}+\epsilon_{i}+\epsilon_{i}^{2}<\sigma_{i-1}, 1 \leq i \leq m$;

(2) $v(z)<\omega(z), 1 \leq k \leq N, z \in \bar{D}$; and

(3) if $F_{i}, 1 \leq i \leq m$, is the union of all connected components of the set $\left\{v \leq \sigma_{i}+\epsilon_{i}\right\}$ that intersect $K_{i}$, then $F_{i} \subset D_{\sigma_{i}+\epsilon_{i}+\epsilon_{i}^{2}}$. 
Let $G_{i}$ be the interior of $F_{i}$. Since $v<\omega$ on $\bar{D}, K_{i} \subset G_{i}$. Clearly, $\bar{G}_{i} \subset F_{i}$.

The set $\left\{v \leq \sigma_{i}+\epsilon_{i}\right\}$ is a semianalytic set and thus has a finite number of connected components intersecting any compact set in $D^{j}$, in particular, $\bar{D}$. The set $F_{i} \subset D$, and therefore it has a neighborhood $U$ where no other components of $\left\{v<\sigma_{i}+\epsilon_{i}\right\}$ are present. This means that $G_{i}$ is an analytic polyhedron.

Let us show that our notion of approximation is stable.

Lemma 3.1. Suppose that an integer $p$ and holomorphic functions $f_{1}, \ldots, f_{N}$ on $D^{j}$ approximate $K$ for $\epsilon>0$. Then there is a $\delta>0$ such that for any holomorphic functions $h_{1}, \ldots, h_{N}$ on $D^{j}$ with the uniform norm $\left\|h_{k}\right\|_{\bar{D}}<\delta$ the functions $g_{k}=$ $f_{k}+h_{k}, 1 \leq k \leq N$, approximate $K$ for $\epsilon$ with the same $p$.

Proof. First, $\left|f_{k}\right|<e^{p \omega}-a$ on $\bar{D}$ for some $a>0$ and every $1 \leq k \leq N$. If $\delta<a$, then $\left|g_{k}\right|<e^{p \omega}$.

We take $\delta$ so small that $1-\delta e^{-p\left(\sigma_{i}+\epsilon_{i}\right)}=e^{-b_{i} p}$, where $0<b_{i}<\epsilon_{i}$ for all $1 \leq i \leq m$. If $z \in \partial G_{i}$, then $\left|f_{k}(z)\right|=e^{p\left(\sigma_{i}+\epsilon_{i}\right)}$ for some $k$. Now

$$
\left|g_{k}(z)\right|>e^{p\left(\sigma_{i}+\epsilon_{i}\right)}\left(1-\delta e^{-p\left(\sigma_{i}+\epsilon_{i}\right)}\right)=e^{p\left(\sigma_{i}+\epsilon_{i}^{\prime}\right)}
$$

where $\epsilon_{i}^{\prime}=\epsilon_{i}-b_{i}$ and $0<\epsilon_{i}^{\prime}<\epsilon_{i}$ for $1 \leq i \leq m$. Hence

$$
v^{\prime}(z)=\sup _{1 \leq k \leq N} \frac{1}{p} \log \left|g_{k}(z)\right|>\sigma_{i}+\epsilon_{i}^{\prime} .
$$

Let $G_{i}^{\prime}$ be the interior of the union $F_{i}^{\prime}$ of connected components of the set $\{z \in$ $\left.D^{j}: v^{\prime}(z) \leq \sigma_{i}+\epsilon_{i}^{\prime}\right\}$ that intersect $K_{i}$. If $F^{\prime}$ is one of the connected components of the set $F_{i}^{\prime}$, then it contains a point of $K_{i}$ and, consequently, intersects a connected component $G$ of $G_{i}$. Since $v^{\prime}>\sigma_{i}+\epsilon_{i}^{\prime}$ on $\partial G_{i}$, the component $G$ must contain $F^{\prime}$. Thus $F_{i}^{\prime} \subset G_{i} \subset F_{i}$. Also, the same reasoning implies that $G_{i}^{\prime} \subset G_{i}$.

There is a positive $\epsilon_{i}^{\prime \prime}<\epsilon_{i}$ such that $F_{i} \subset D_{\sigma_{i}+\epsilon_{i}^{\prime \prime}+\epsilon_{i}^{\prime \prime 2}}$. Let us choose $\delta$ so small that $\epsilon_{i}^{\prime \prime}<\epsilon_{i}^{\prime}$ for all $1 \leq i \leq m$. Then $F_{i}^{\prime} \subset D_{\sigma_{i}+\epsilon_{i}^{\prime}+\epsilon_{i}^{\prime}}$, and the proof is complete.

The following lemma shows the existence of holomorphic approximations.

Lemma 3.2. For any sufficiently small $\epsilon>0$ and any integer $j$ there exist a positive integer $p$ and holomorphic functions $f_{1}, \ldots, f_{N}$ on $D^{j}$ that approximate $K$ on $\bar{D}$ for $\epsilon$.

Proof. We assume that $\sigma_{i}+\epsilon+\epsilon^{2}<\epsilon$ when $1 \leq i \leq m$. Let us take $0<\delta<$ $\min \left\{\epsilon^{2} / 2,-\sigma_{1}\right\}$ and $a>0$ so big that $a \phi<\omega$ on $\bar{D}_{-\delta}$. Then the function $\omega^{\prime}=$ $\max \{\omega, a \phi\}-\delta$ on $\bar{D}$ and $a \phi-\delta$ on $D^{j} \backslash D$ is plurisubharmonic on $D^{j}$. By Bremermann's approximation theorem (see [S] for a proof) there exist a positive integer $p$ and holomorphic functions $f_{1}, \ldots, f_{N}$ on $D^{j}$ such that

$$
\omega^{\prime}(z)<v(z)=\sup _{1 \leq k \leq N} \frac{1}{p} \log \left|f_{k}(z)\right|<\omega^{\prime}(z)+\delta
$$

on $D^{2 j}$. But $\omega^{\prime}=\omega-\delta$ on $D_{-\delta}$. Hence $\omega-\delta<v<\omega$ on $D_{-\delta}$. Since $v<0$ on $\partial D$ and $v<-\delta$ on $\partial D_{-\delta}$, we see that $v<\omega$ on $\bar{D} \backslash D_{-\delta}$. Since $v>-\delta$ on $\bar{D} \backslash D_{-\delta}$, we see that $\omega(z)-\epsilon^{2}<v(z)<\omega(z)$ on $\bar{D}$.

By the latter inequality the set $A=\left\{v \leq \sigma_{i}+\epsilon\right\}$ belongs to $D_{\sigma_{i}+\epsilon+\epsilon^{2}}$. Hence for every $1 \leq i \leq m$ the union $F_{i}$ of all connected components of $A$ that intersect $K_{i}$ also belongs to $D_{\sigma_{i}+\epsilon+\epsilon^{2}}$. So $p$ and $f_{1}, \ldots, f_{N}$ approximate $K$ for $\epsilon$. 
The following theorem approximates any condenser by $n$ holomorphic functions.

Theorem 3.3. For any sufficiently small $\epsilon>0$ and any sufficiently large integer $j$ there exist an integer $p$ and $n$ holomorphic functions $f_{1}, \ldots, f_{n}$ on $D^{j}$ that approximate a pluriregular condenser $K$ for $\epsilon$ and the system of equations $f_{1}=\cdots=f_{n}=0$ has only simple roots in $D$.

Proof. Suppose that $N>n$ is the minimal number of holomorphic functions $f_{1}, \ldots, f_{N}$ on any $D^{j}$ that approximate $K$ for $\epsilon$ with some $p$. Take any such approximation. By Lemma 3.1 it is stable for some $\delta>0$. Note that none of the chosen functions is equal identically to zero. As in the proof of Lemma 4 in $[\mathrm{B}$. (see also Lemma 7B1 in [GR]), we can find holomorphic functions $h_{2}, \ldots, h_{N}$ on $D^{j+1}$ such that $\left\|h_{k}\right\|_{\bar{D}^{2 j}}<\delta$ and the mapping $\left(f_{2} f_{1}^{-1}+h_{2}, \ldots, f_{N} f_{1}^{-1}+h_{N}\right)$ is light, i.e., it has zero-dimensional preimages of values of every point on $D^{2 j}$. Since $\left|f_{1}\right|<1$ on $\bar{D}$, by Lemma 3.1 the functions $g_{k}=f_{k}+h_{k} f_{1}$ for $k=2, \ldots, N$ and $g_{1}=f_{1}$ approximate $K$ for $\epsilon$ with the same $p$.

Let us replace the functions $f_{i}$ by the functions $g_{i}$ preserving notation, i.e., $f_{i}:=g_{i}$. In the terminology of [B] and [GR the analytic polyhedra $G_{i}$ are now prepared. We want to show that for a sufficiently large integer $q$ there is a number $p^{\prime}$ so that $p^{\prime}$ and the functions $f_{k}^{q}-f_{1}^{q}, 2 \leq k \leq N$ approximate $K$ for $\epsilon$. The proof follows the steps of Theorem 2 in [B] or Lemma 7B2 in GR.

There are $a<1$ and $j_{1}>j$ such that $\left|f_{k}\right| \leq a$ on $\bar{D}^{j_{1}}$ for all $1 \leq k \leq N$. Hence $\left|f_{k}^{q}-f_{1}^{q}\right| \leq 1$ on $D^{j_{1}}$ when $q$ is greater then some $q_{0}$. Also on $K_{i}$,

$$
\left|f_{k}^{q}-f_{1}^{q}\right| \leq 2 e^{p q \sigma_{i}}<e^{\left(p q+\ln 2 / \sigma_{i}\right) \sigma_{i}}<e^{p^{\prime} \sigma_{i}},
$$

where $p^{\prime}=p q-\mu$ and $\mu$ is the least integer greater than $-\ln 2 / \sigma_{i}$ for all $1 \leq i \leq m$. Note that $\mu \geq 0$. We assume that $q \geq q_{1} \geq q_{0}$, so that $p^{\prime}>0$ for all $i$. Thus $\left|f_{k}^{q}-f_{1}^{q}\right|<e^{p^{\prime} \omega}$ on $\bar{D}$ when $q \geq q_{1}$.

Let us show that $\bar{D}_{\sigma_{i}+\epsilon_{i}} \subset G_{i}$. Since $\sigma_{i}+\epsilon_{i}<\sigma_{i-1}$, the set $\bar{D}_{\sigma_{i}+\epsilon_{i}}$ belongs to the interior $V_{i-1}$ of $K_{i-1}$. The set $\bar{G}_{i}$ belongs to $D_{\sigma_{i}+\epsilon_{i}+\epsilon_{i}^{2}}$, which in its turn belongs to $V_{i-1}$ because $\sigma_{i}+\epsilon_{i}+\epsilon_{i}^{2}<\sigma_{i-1}$. The set $G_{i}$ contains $K_{i}$ and, therefore, the function $\omega$ is maximal on $V_{i-1} \backslash \bar{G}_{i}$. The boundary of $V_{i-1} \backslash \bar{G}_{i}$ consists of the boundary of $V_{i-1}$, where $\omega=\sigma_{i-1}$, and the boundary of $G_{i}$, where $\omega>v=\sigma_{i}+\epsilon_{i}$. By the maximality of $\omega$ on $V_{i-1} \backslash \bar{G}_{i}$, the function $\omega>\sigma_{i}+\epsilon_{i}$ there. Hence $\bar{D}_{\sigma_{i}+\epsilon_{i}} \subset \bar{G}_{i}$. But $\omega>\sigma_{i}+\epsilon_{i}$ on $\partial G_{i}$ and, consequently, $\bar{D}_{\sigma_{i}+\epsilon_{i}} \subset G_{i}$.

Hence we can find positive $\epsilon_{i}^{\prime}<\epsilon_{i}$ such that

$$
\bar{D}_{\sigma_{i}+\epsilon_{i}^{\prime}} \subset G_{i} \subset \bar{G}_{i} \subset F_{i} \subset D_{\sigma_{i}+\epsilon_{i}^{\prime}+\epsilon_{i}^{\prime 2}}
$$

for all $1 \leq i \leq m$.

Let us take an open set $U_{i} \subset \subset D_{\sigma_{i}+\epsilon_{i}^{\prime}+\epsilon_{i}^{\prime 2}}$ such that the domain $G_{i}$ is a prepared analytic polyhedron with the frame $\left(U_{i}, g_{1 i}, \ldots, g_{N i}\right)$, where $g_{k i}=e^{-p\left(\sigma_{i}+\epsilon_{i}\right)} f_{k}$, i.e., $G_{i}=\left\{z \in U_{i}:\left|g_{k i}(z)\right|<1,1 \leq k \leq N\right\}$ (see [B] or [GR] for the terminology). We also take an open set $U_{i}^{\prime} \subset \subset U_{i}$ containing $\bar{G}_{i}$. Let $V_{i}=U_{i}^{\prime} \backslash \bar{D}_{\sigma_{i}+\epsilon_{i}^{\prime}}$ be a neighborhood of $\partial G_{i}$. This neighborhood is relatively compact in $U_{i}$. Since $\omega=$ $\sigma_{i}+\epsilon_{i}^{\prime}$ on $\partial V_{i} \cap G_{i}=\partial D_{\sigma_{i}+\epsilon_{i}^{\prime}}$ and $\left|f_{k}\right|<e^{p \omega}$, we see that $\left|g_{k i}\right|<r_{i}^{-1}=e^{p\left(\epsilon_{i}^{\prime}-\epsilon_{i}\right)}<1$ on $\partial V_{i} \cap G_{i}$ for all $1 \leq k \leq N$. By Theorem 2 of [B] or Lemma 7B2 from [GR], there is a sufficiently large $q_{2} \geq q_{1}$ such that for all $q \geq q_{2}$ and all $1 \leq i \leq m$ the union $R_{q}^{i}$ of $\bar{D}_{\sigma_{i}+\epsilon_{i}^{\prime}}$ and all connected components of the set

$$
\left\{z \in \bar{V}_{i}: r_{i}^{q}\left|g_{k i}^{q}-g_{N i}^{q}\right|<1,2 \leq k \leq N\right\}
$$


that intersect $\bar{D}_{\sigma_{i}+\epsilon_{i}^{\prime}}$ is a polyhedron with the frame

$$
\left(U_{i}^{\prime} \backslash \bar{D}_{\sigma_{i}+\epsilon_{i}^{\prime}}, r_{i}^{q}\left(g_{2 i}^{q}-g_{1 i}^{q}\right), \ldots, r_{i}^{q}\left(g_{N i}^{q}-g_{1 i}^{q}\right)\right) .
$$

Recall that $p^{\prime}=p q-\mu, \mu \geq 0$, and the function

$$
v^{\prime}=\sup _{2 \leq k \leq N} \frac{1}{p^{\prime}} \log \left|f_{k}^{q}-f_{1}^{q}\right|<\omega
$$

on $\bar{D}$. It is easy to verify that $p q\left(\sigma_{i}+\epsilon_{i}^{\prime}\right)>p^{\prime}\left(\sigma_{i}+\epsilon_{i}^{\prime \prime}\right)$ when

$$
\epsilon_{i}^{\prime \prime}<\epsilon_{i}^{\prime}+\frac{\mu\left(\sigma_{i}+\epsilon_{i}^{\prime}\right)}{p^{\prime}} \leq \epsilon_{i}^{\prime} \text {. }
$$

We take $q_{3} \geq q_{2}$ and $\epsilon_{i}^{\prime \prime}$ such that $\bar{U}_{i}^{\prime} \subset D_{\sigma_{i}+\epsilon_{i}^{\prime \prime}+\epsilon_{i}^{\prime \prime 2}}$ for all $q \geq q_{3}$ and all $i$.

Let $F^{\prime}$ be a connected component of the set $F_{i}^{\prime}=\left\{v^{\prime} \leq \sigma_{i}+\epsilon_{i}^{\prime \prime}\right\}$ intersecting $K_{i}$. Let us show that $F^{\prime} \subset G_{i}$. If $z_{0} \in F^{\prime} \cap K_{i}$, then

$$
\left|g_{k i}^{q}\left(z_{0}\right)-g_{1}^{q}\left(z_{0}\right)\right|=e^{-p q\left(\sigma_{i}+\epsilon_{i}\right)}\left|f_{k}^{q}\left(z_{0}\right)-f_{1}^{q}\left(z_{0}\right)\right| \leq e^{-p q\left(\sigma_{i}+\epsilon_{i}\right)} e^{p^{\prime} \sigma_{i}}<1 .
$$

Thus $z_{0}$ belongs to one of the components $R$ of the set $R_{q}^{i}$. If $z_{1} \in \partial R$, then $\left|g_{k i}^{q}\left(z_{1}\right)-g_{1}^{q}\left(z_{1}\right)\right|=1$ for some $k$. Hence

$$
\left|f_{k}^{q}\left(z_{1}\right)-f_{1}^{q}\left(z_{1}\right)\right|=e^{p q\left(\sigma_{i}+\epsilon_{i}\right)}>e^{p^{\prime}\left(\sigma_{i}+\epsilon_{i}^{\prime \prime}\right)},
$$

or $v^{\prime}\left(z_{1}\right)>\sigma_{i}+\epsilon_{i}^{\prime \prime}$. Therefore $F^{\prime} \subset R \subset R_{q}^{i}$. Since $R_{q}^{i} \subset U_{i}^{\prime} \subset D_{\sigma_{i}+\epsilon_{i}^{\prime \prime}+\epsilon_{i}^{\prime \prime 2}}$, we see that the functions $f_{k}^{q}-f_{1}^{q}, 2 \leq k \leq N$, approximate $K$ for $\epsilon$.

So $N=n$. Let us show that the new functions can be corrected so that the system $f_{1}=\cdots=f_{n}=0$ has only simple zeros.

Suppose that the system $f_{1}=\cdots=f_{n}=0$ has non-simple zeros. The Jacobian of the mapping $f=\left(f_{1}, \ldots, f_{n}\right)$ is not identically zero, because otherwise through every point of $D$ there would pass a complex curve where $v$ is constant. But $v<\sigma_{1}$ on $K_{1}$ and $v=\sigma_{1}+\epsilon_{1}$ on $\partial G_{1}$.

Our approximation is stable for some $\delta>0$. By Sard's theorem there exists a point $\left(c_{1}, \ldots, c_{n}\right)$ in $\mathbb{C}^{n}$ such that $f$ is non-degenerate at all preimages of this point and $\left|c_{k}\right|<\delta$ for all $k$. Let $g_{i}=f_{i}-c_{i}$. The system $g_{1}=\cdots=g_{n}=0$ has only simple zeros in $D$, and approximates $K$ for $\epsilon$ with the same $p$.

\section{Approximation of COndensers by Green functions}

The following lemma uses the existence of holomorphic approximations of relative extremal functions of pluriregular condensers to obtain an approximation of some sort of extremal functions by Green functions with the controlled behavior of Monge-Ampère masses.

Lemma 4.1. Let $K=\left(K_{1}, \ldots, K_{m}, \sigma_{1}, \ldots, \sigma_{m}\right)$ be a pluriregular condenser in a strictly hyperconvex domain $D \subset \mathbb{C}^{n}$. Then there are sequences of positive numbers $\delta_{j}$ converging to zero, Green functions $g_{j}$ on $D$, and, for every $1 \leq i \leq m$, numbers $\sigma_{i j}^{\prime}<\sigma_{i}$ converging to $\sigma_{i}$ and open sets $V_{i j}$ and $W_{i j}\left(W_{m j}=\emptyset\right)$ such that

$$
D_{\sigma_{i j}^{\prime}} \subset \subset W_{i j} \subset \subset D_{\sigma_{i}} \subset \subset V_{i j} \subset \subset D_{\sigma_{i j}^{\prime}},
$$


$g_{j}>\sigma_{i}$ on $\partial V_{i j}, g_{j}>\sigma_{i j}^{\prime}$ on $\partial W_{i j}$, the poles of $g_{j}$ lie in the union of the sets $Z_{i j}=V_{i j} \backslash \bar{W}_{i j}, 1 \leq i \leq m$, and

$$
\int_{Z_{i j}}\left(d d^{c} \omega\right)^{n}+\delta_{j} \geq \int_{Z_{i j}}\left(d d^{c} g_{j}\right)^{n} \geq \int_{Z_{i j}}\left(d d^{c} \omega\right)^{n}-\delta_{j} .
$$

Proof. For every $1 \leq i \leq m$ let us choose an increasing sequence of numbers $\sigma_{i j}^{\prime}$ lying strictly between $\sigma_{i+1}$ and $\sigma_{i}$ and converging to $\sigma_{i}$. We set $K_{2 i-1, j}=K_{i}$ and $K_{2 i, j}=\bar{D}_{\sigma_{i j}^{\prime}}$. Let $\sigma_{2 i-1, j}=\sigma_{i}$ and $\sigma_{2 i, j}=\sigma_{i j}^{\prime}$. We introduce the pluriregular condenser $K^{j}$ formed by a system of compact sets $K_{i j}$ and numbers $\sigma_{i j}$. Note that $\omega(z, K, D)=\omega\left(z, K^{j}, D\right)$ for all $j$.

For every $j$ we choose a sequence of systems of holomorphic functions $f_{1 j}, \ldots, f_{n j}$ and integers $p_{j}$ that approximate $K^{j}$ for $\epsilon_{j}<1 / j$. We assume that the systems $f_{1 j}=\cdots=f_{n j}=0$ have only simple roots and the numbers $\epsilon_{j}$ are so small that

$$
a_{j}=\frac{\sigma_{i j}-\sigma_{i-1, j}+\epsilon_{j}}{\sigma_{i j}-\sigma_{i-1, j}+\epsilon_{j}+\epsilon_{j}^{2}}<1+\frac{1}{j}
$$

for all $1 \leq i \leq 2 m$. Let

$$
v_{j}=\sup _{1 \leq k \leq n} \frac{1}{p_{j}} \log \left|f_{k j}\right| .
$$

We will add an index $j$ to all parameters of these approximations so that $\sigma_{i j}+$ $\epsilon_{i j}+\epsilon_{i j}^{2}<\sigma_{i-1, j}$

$$
K_{i j} \subset G_{i j} \subset \bar{G}_{i j} \subset D_{\sigma_{i j}+\epsilon_{i j}+\epsilon_{i j}^{2}}
$$

and $v_{j}=\sigma_{i j}+\epsilon_{i j}$ on $\partial G_{i j}$.

Let $\omega_{i j}$ and $v_{i j}$ be the auxiliary functions $\left(1-\epsilon_{i j}\right)\left(\omega-\sigma_{i j}-\epsilon_{i j}-\epsilon_{i j}^{2}\right)$ and $v_{j}-\sigma_{i j}-\epsilon_{i j}$ respectively. On $\partial G_{i j}$ the functions $v_{i j}=0$ and $\omega_{i j}<0$. Since $\omega \leq \sigma_{i j}$ on $K_{i j}$ and $v_{j}<\omega$ on $D$, we see that

$$
\omega_{i j}=\omega-\sigma_{i j}-\epsilon_{i j}-\epsilon_{i j}\left(\omega-\sigma_{i j}-\epsilon_{i j}^{2}\right)>v_{i j}
$$

there. Thus the set $F_{i j}=\left\{v_{i j}<\omega_{i j}\right\} \cap G_{i j}$ contains $K_{i j}$ and compactly belongs to $G_{i j}$. By the Comparison Principle,

$$
\int_{G_{i j}}\left(d d^{c} v_{j}\right)^{n} \geq \int_{F_{i j}}\left(d d^{c} v_{i j}\right)^{n} \geq \int_{F_{i j}}\left(d d^{c} \omega_{i j}\right)^{n} .
$$

By the maximality of $\omega_{i j}$ on $G_{i j} \backslash K_{i j}$ we get

$$
\int_{G_{i j}}\left(d d^{c} v_{j}\right)^{n} \geq \int_{G_{i j}}\left(d d^{c} \omega_{i j}\right)^{n}=\left(1-\epsilon_{j}\right) \int_{G_{i j}}\left(d d^{c} \omega\right)^{n} .
$$

Now we take the set $P_{i j}$ of those poles of $v_{j}$ that lie in $G_{i j}$ and introduce the Green function $g_{i j}$ on $D_{\sigma_{i-1, j}}$ with poles in $P_{i j}$ of weight $1 / p_{j}$. The functions $g_{i j}$ have poles of the same weight as $v_{j}$ in $P_{i j}$, and $g_{i j}=0$ on $\partial D_{\sigma_{i-1, j}}$. Hence $g_{i j}>v_{j}-\sigma_{i-1, j}$ on $\bar{D}_{\sigma_{i-1, j}}$.

Let $\omega_{i j}^{\prime}$ be the restriction of $a_{j}\left(\omega-\sigma_{i-1, j}\right)$ to $D_{\sigma_{i-1, j}}$, and let $v_{i j}^{\prime}=\max \left\{g_{i j}, \sigma_{i j}-\right.$ $\left.\sigma_{i-1, j}+\epsilon_{i j}\right\}$. Then $\omega_{i j}^{\prime}=v_{i j}^{\prime}=0$ on $\partial D_{\sigma_{i-1, j}}$ and $\omega_{i j}^{\prime}<v_{i j}^{\prime}$ on $G_{i j}$, because $\omega_{i j}^{\prime}<\sigma_{i j}-\sigma_{i-1, j}+\epsilon_{i j}$ there. Since the function $g_{i j}>\sigma_{i j}-\sigma_{i-1, j}+\epsilon_{i j}$ on $\partial G_{i j}$ and 
is maximal on $D_{\sigma_{i-1, j}} \backslash \bar{G}_{i j}$, we see that $\omega_{i j} \leq v_{i j}$ on $D_{\sigma_{i-1, j}}$. By the Comparison Principle,

By (2.1),

$$
\int_{D_{\sigma_{i-1, j}}}\left(d d^{c} v_{i j}^{\prime}\right)^{n} \leq a_{j} \int_{D_{\sigma_{i-1, j}}}\left(d d^{c} \omega\right)^{n} .
$$

$$
\int_{D_{\sigma_{i-1, j}}}\left(d d^{c} v_{i j}^{\prime}\right)^{n}=\int_{D_{\sigma_{i-1, j}}}\left(d d^{c} g_{i j}\right)^{n}
$$

By the maximality of $g_{i j}$ and $\omega$ on $D_{\sigma_{i-1, j}} \backslash \bar{G}_{i j}$ we get

$$
\int_{G_{i j}}\left(d d^{c} v_{j}\right)^{n}=\int_{G_{i j}}\left(d d^{c} g_{i j}\right)^{n} \leq a_{j} \int_{G_{i j}}\left(d d^{c} \omega\right)^{n} .
$$

Since

by (4.1) and (4.2)

$$
\int_{G_{2 i, j}}\left(d d^{c} \omega\right)^{n}=\int_{G_{2 i+1, j}}\left(d d^{c} \omega\right)^{n}
$$

$$
\int_{G_{2 i, j}}\left(d d^{c} v_{j}\right)^{n}-\int_{G_{2 i+1, j}}\left(d d^{c} v_{j}\right)^{n} \leq \frac{2}{j} \int_{G_{2 i+1, j}}\left(d d^{c} \omega\right)^{n} .
$$

Now for $1 \leq i \leq m$ we let $V_{i j}=G_{2 i-1, j}$ and $W_{i j}=G_{2 i, j}$. For each $j$ let us consider the Green function $g_{j}$ on $D$ with poles of weight $1 / p_{j}$ at those poles of $v_{j}$ that lie in the union of the sets $G_{2 m-1}$ and $G_{2 i-1, j} \backslash \overline{G_{2 i, j}}, 1 \leq i \leq m-1$. By the definition of $g_{j}$ and (4.2),

$$
\int_{V_{i j}}\left(d d^{c} g_{j}\right)^{n} \leq \int_{V_{i j}}\left(d d^{c} v_{j}\right)^{n} \leq\left(1+\frac{1}{j}\right) \int_{V_{i j}}\left(d d^{c} \omega\right)^{n} .
$$

Now

$$
\int_{V_{i j}}\left(d d^{c} g_{j}\right)^{n}=\int_{V_{i j}}\left(d d^{c} v_{j}\right)^{n}-\sum_{k=i}^{m-1} \int_{G_{2 k, j} \backslash \bar{G}_{2 k+1, j}}\left(d d^{c} v_{j}\right)^{n} .
$$

By (4.1) and (4.3),

$$
\int_{V_{i j}}\left(d d^{c} g_{j}\right)^{n} \geq\left(1-\frac{2 m}{j}\right) \int_{V_{i j}}\left(d d^{c} \omega\right)^{n} .
$$

Let $\rho_{j}=2 m / j$ and

$$
\delta_{j}=2 \rho_{j} \int_{D}\left(d d^{c} \omega\right)^{n} .
$$

By the above inequalities,

$$
\begin{aligned}
& \int_{Z_{i j}}\left(d d^{c} g_{j}\right)^{n}=\int_{V_{i j}}\left(d d^{c} g_{j}\right)^{n}-\int_{V_{i+1, j}}\left(d d^{c} g_{j}\right)^{n} \\
& \leq\left(1+\delta_{j}\right) \int_{V_{i j}}\left(d d^{c} \omega\right)^{n}-\left(1-\delta_{j}\right) \int_{V_{i+1, j}}\left(d d^{c} \omega\right)^{n} \leq \int_{Z_{i j}}\left(d d^{c} \omega\right)^{n}+\delta_{j} .
\end{aligned}
$$


In the same way,

$$
\begin{aligned}
\int_{Z_{i j}}\left(d d^{c} g_{j}\right)^{n} & \geq\left(1-\delta_{j}\right) \int_{V_{i j}}\left(d d^{c} \omega\right)^{n}-\left(1+\delta_{j}\right) \int_{V_{i+1, j}}\left(d d^{c} \omega\right)^{n} \\
& \geq \int_{Z_{i j}}\left(d d^{c} \omega\right)^{n}-\delta_{j} .
\end{aligned}
$$

Since $g_{j}>v_{j}$ on $D$, we see that $g_{j}>\sigma_{i}$ on $\partial V_{i j}$ and $g_{j}>\sigma_{i j}^{\prime}$ on $\partial W_{i j}$. The lemma is proved.

The following lemma shows that a sequence of Green functions obtained in the previous result approximates $\omega$ in the more usual sense. For a simple pluriregular condenser the lemma was proved in $[\mathrm{NP}]$.

Lemma 4.2. Suppose that a sequence of Green functions $g_{j}$ on $D$ satisfies the conditions of Lemma 4.1. Then this sequence converges to $\omega(z)=\omega(z, K, D)$ uniformly on compacta in $D_{\sigma_{i-1}} \backslash K_{i}, 1 \leq i \leq m$. Moreover, if $\psi$ is a continuous function on $\mathbb{R}$, then

$$
\lim _{j \rightarrow \infty} \int_{D} \psi(\omega(z))\left(d d^{c} g_{j}\right)^{n}=\int_{D} \psi(\omega(z))\left(d d^{c} \omega\right)^{n} .
$$

Proof. Let us denote by $g_{i j}$ the restriction of $g_{j}$ to the open set $Z_{i j}$. Since $g_{j}>$ $\sigma_{i}>\sigma_{i j}^{\prime}$ on $\partial V_{i j}$ and $g_{j}>\sigma_{i j}^{\prime}$ on $\partial W_{i j}$, the set $\left\{g_{i j}<\sigma_{i j}^{\prime}-\delta_{j}\right\}$ compactly belongs to $Z_{i j}$. Let $g_{i j}^{\prime}=\max \left\{g_{i j}, \sigma_{i j}^{\prime}-\delta_{j}\right\}$. Then the function $g_{j}^{\prime}$ defined as $g_{i j}^{\prime}$ on $Z_{i j}$, $1 \leq i \leq m$, and $g_{j}$ otherwise is plurisubharmonic on $D$. Moreover, by (2.1),

$$
\int_{Z_{i j}}\left(d d^{c} g_{j}^{\prime}\right)^{n}=\int_{Z_{i j}}\left(d d^{c} g_{j}\right)^{n} .
$$

Let

Clearly, $\alpha_{j}$ converges to 1 .

$$
\alpha_{j}=\min \left\{\frac{\sigma_{i j}^{\prime}}{\sigma_{i j}^{\prime}-\delta_{j}}: 1 \leq i \leq m\right\} .
$$

Let $v_{j}=\alpha_{j} g_{j}^{\prime}$. Since $v_{j} \geq \sigma_{i j}^{\prime}>\omega$ on every $Z_{i j}$, by the maximality of $v$ outside of the union of the $Z_{i j}$ it is greater than $\omega$ on $D$. Therefore, integrating by parts, we get

$$
\begin{aligned}
& \int_{D}(-\omega)\left(d d^{c} \omega\right)^{n} \geq \int_{D}\left(-v_{j}\right)\left(d d^{c} \omega\right)^{n}=\int_{D}(-\omega) d d^{c} v_{j} \wedge\left(d d^{c} \omega\right)^{n-1} \\
& \geq \int_{D}\left(-v_{j}\right) d d^{c} v_{j} \wedge\left(d d^{c} \omega\right)^{n-1}=\cdots \geq \int_{D}\left(-v_{j}\right)\left(d d^{c} v_{j}\right)^{n} .
\end{aligned}
$$

Thus

$$
\begin{aligned}
0 \leq & \int_{D}\left(v_{j}-\omega\right)\left(d d^{c} \omega\right)^{n} \leq \int_{D} v_{j}\left(d d^{c} v_{j}\right)^{n}-\int_{D} \omega\left(d d^{c} \omega\right)^{n} \\
& =\sum_{i=1}^{m}\left(\int_{Z_{i j}} v_{j}\left(d d^{c} v_{j}\right)^{n}-\int_{Z_{i j}} \omega\left(d d^{c} \omega\right)^{n}\right)
\end{aligned}
$$


The support of $\left(d d^{c} \omega\right)^{n}$ lies in the boundaries of the $K_{i}$, where $\omega=\sigma_{i}$. The support of $\left(d d^{c} v_{j}^{\prime}\right)^{n}$ lies where $g_{j}^{\prime}=\sigma_{i j}^{\prime}-\delta_{j}$. Thus, by the previous inequality,

$$
0 \leq \int_{D}\left(v_{j}-\omega\right)\left(d d^{c} \omega\right)^{n} \leq \sum_{i=1}^{m}\left(\alpha_{j}^{n} \sigma_{i j}^{\prime} \int_{Z_{i j}}\left(d d^{c} g_{j}^{\prime}\right)^{n}-\sigma_{i} \int_{Z_{i j}}\left(d d^{c} \omega\right)^{n}\right) .
$$

By (2.1) and the integral inequality in Lemma 4.1 ,

$$
\int_{Z_{i j}}\left(d d^{c} g_{j}^{\prime}\right)^{n}=\int_{Z_{i j}}\left(d d^{c} g_{j}\right)^{n} \geq \int_{Z_{i j}}\left(d d^{c} \omega\right)^{n}-\delta_{j}
$$

Hence

$$
0 \leq \int_{D}\left(v_{j}-\omega\right)\left(d d^{c} \omega\right)^{n} \leq \sum_{i=1}^{m}\left(\left(\alpha_{j}^{n} \sigma_{i j}^{\prime}-\sigma_{i}\right) \int_{Z_{i j}}\left(d d^{c} \omega\right)^{n}-\delta_{j} \alpha_{j}^{n} \sigma_{i j}^{\prime}\right) .
$$

Therefore,

$$
\lim _{j \rightarrow \infty} \int_{\left\{v_{j}-\omega>a\right\}}\left(d d^{c} \omega\right)^{n}=0
$$

for every $a>0$.

We fix some $\delta>0$ and choose $\epsilon>0$ such that $\epsilon|z|^{2}<\delta / 2$ on $D$, and we let $u_{j}=v_{j}+\epsilon|z|^{2}-\delta$.

Note that

$$
\left(d d^{c}\left(\epsilon|z|^{2}-\delta\right)\right)^{n}=\epsilon^{n} c_{n} d V,
$$

where the constant $c_{n}$ depends only on $n$, and $d V$ is the volume form. Let $E_{j}=$ $\left\{z \in D: \omega<u_{j}\right\}$. Since $u_{j}<-\delta / 2$ on $\partial D$ and $v_{j}-\omega>\delta / 2$ on $E_{j}$, the set $E_{j}$ compactly belongs to $D$ and $E_{j} \subset\left\{v_{j}-\omega>\delta / 2\right\}$. By the subadditivity of the Monge-Ampère operator and the Comparison Principle we have

or

$$
\int_{E_{j}}\left(d d^{c} v_{j}\right)^{n}+\int_{E_{j}}\left(d d^{c} \epsilon|z|^{2}-\delta\right)^{n} \leq \int_{E_{j}}\left(d d^{c} u_{j}\right)^{n} \leq \int_{E_{j}}\left(d d^{c} \omega\right)^{n}
$$

$$
\epsilon^{n} c_{n} m\left(E_{j}\right) \leq \int_{\left\{v_{j}-\omega>\delta / 2\right\}}\left(d d^{c} \omega\right)^{n}
$$

Thus

$$
\lim _{j \rightarrow \infty} m\left(E_{j}\right)=0 .
$$

The set $F_{j}=\left\{\omega<v_{j}-\delta\right\} \subset E_{j}$. Let us take $r>0$ such that $|\omega(z)-\omega(w)|<\delta$ when $|z-w|<r$, and take $j_{0}$ such that $m\left(E_{j}\right)<\delta m(B(z, r))$ for all $j \geq j_{0}$. If $B=B\left(z_{0}, r\right) \in D$, then

$$
\begin{aligned}
& v_{j}\left(z_{0}\right) \leq \frac{1}{m(B)} \int_{B} v_{j}(z) d V=\frac{1}{m(B)}\left(\int_{B \backslash F_{j}} v_{j} d V+\int_{B \cap F_{j}} v_{j} d V\right) \\
& \leq \frac{1}{m(B)}\left(\int_{B}(\omega+\delta) d V-\int_{B \cap F_{j}}(\omega+\delta) d V\right) \leq \omega\left(z_{0}\right)+\left(2-\sigma_{m}\right) \delta .
\end{aligned}
$$


Since $v_{j}(z) \geq \omega(z)$, we see that the functions $v_{j}$ converge to $\omega$ uniformly on $D$. Consequently, the functions $g_{j}$ converge to $\omega$ uniformly on compacta in $D_{\sigma_{i-1}} \backslash K_{i}$, $1 \leq i \leq m$.

The last statement of the lemma follows immediately from the integral inequality in Lemma 4.1

\section{Approximation of PLURisubharmonic FUnCtions}

Theorem 5.1. If $D$ is a strongly hyperconvex domain in $\mathbb{C}^{n}$ with an exhaustion function $\phi$ and $u$ is a negative plurisubharmonic function on $D$ with zero boundary values, then there is a sequence of pluricomplex multipole Green functions $g_{j}$ converging to $u$ in $L^{1}(D)$. Moreover, if $u$ is continuous on $D$ and a function $\psi \in C_{0}((-\infty, 0])$, then

$$
\lim _{j \rightarrow \infty} \int_{D} \psi(u(z))\left(d d^{c} g_{j}\right)^{n}=\int_{D} \psi(u(z))\left(d d^{c} u\right)^{n} .
$$

Proof. We start to prove this theorem for a continuous plurisubharmonic function $u$ on $D$ with zero boundary values, for which there is an open set $D^{\prime} \subset \subset D$ such that $\partial D^{\prime}$ is a smooth hypersurface, $u$ is equal to $\sigma_{1}<0$ on $\partial D^{\prime}$, maximal on $D \backslash \bar{D}^{\prime}$ and is of class $C^{2}$ and strictly plurisubharmonic in $D^{\prime}$. Then $u$ has finitely many local minima $z_{1}, \ldots, z_{p}$ in $D^{\prime}$.

By Sard's theorem, for every $j \geq 1$ we can find numbers $\sigma_{m_{j} j}<\sigma_{m_{j}-1, j}<\cdots<$ $\sigma_{2 j}<\sigma_{1 j}=\sigma_{1}<\sigma_{0}=0$ such that:

(1) $\sigma_{i j}-\sigma_{i+1, j}<1 / j$ and the function $u$ is not degenerate on $\left\{u=\sigma_{i j}\right\}$, $1 \leq i \leq m_{j}$

(2) if $z_{k}$ is a local minimum of $u$ and $\sigma_{i j}<u\left(z_{k}\right)<\sigma_{i-1, j}$, then the connected component of the set $\left\{u<\sigma_{i-1, j}\right\}$ that contains $z_{k}$ belongs to a ball $B_{k j}$ and $\sum_{k=1}^{p} m\left(B_{k j}\right)<1 / j$.

By the first condition the sets $K_{i j}=\left\{u \leq \sigma_{i j}\right\}$ have smooth boundaries and, therefore, are pluriregular. So the condenser $K_{j}$ formed by the $K_{i j}$ and $\sigma_{i j}, 0 \leq$ $i \leq m_{j}$, has the continuous relative extremal function $\omega_{j}$.

For every $\omega_{j}$ we take a sequence of Green functions $g_{j m}$ provided by Lemma 4.2 and select a subsequence $g_{j q_{m}}$ that converges in $L_{l o c}^{1}(D)$ to a plurisubharmonic function $v_{j}$ or $-\infty$. But it cannot converge to $-\infty$, and, because it converges to $\omega_{j}$ uniformly on compacta in $\bar{D} \backslash \bar{D}^{\prime}$, it converges to $v_{j}$ in $L^{1}(D)$. For every $i$ the function $v_{j}$ coincides with $\omega_{j}$ on the sets $\left\{\omega_{j}<\sigma_{i-1, j}\right\} \backslash K_{i j}$ or everywhere except the sets $\left\{\omega_{j}=\sigma_{i j}\right\}$. A set $\left\{\omega_{j}=\sigma_{i j}\right\}$ either is a smooth surface or contains a local minimum $z_{k}$. In the first case $v_{j}=\omega_{j}$ on this set. In the second case the set belongs to the ball $B_{k j}$.

Let $A$ be the infimum of $u$ on $D$. There is a point in a ball $B_{k j}$ where $v_{j}(z)=$ $\omega_{j}(z) \geq A$. If $B_{k j}^{\prime}$ is a ball centered at that point and of radius twice the radius of $B_{k j}$, then

$$
\sum_{k=1}^{p} \int_{B_{k j}^{\prime}} v_{j} d V \geq \sum_{k=1}^{p} A m\left(B_{k j}^{\prime}\right) \geq \frac{A}{j}
$$

Thus

$$
\int_{D}\left|v_{j}-\omega_{j}\right| d V \leq \frac{2 A}{j} .
$$


Since the functions $\omega_{j}$ converge uniformly to $u$, the functions $v_{j}$ converge to $u$ in $L^{1}(D)$ and the functions $\left(d d^{c} \omega_{j}\right)^{n}$ weak-* converge to $\left(d d^{c} u\right)^{n}$.

Let us take a countable dense set of functions $\left\{\psi_{q}\right\}$ in $C_{0}((-\infty, 0])$. Since there is a sequence of $g_{j p_{j}}$ that converges to $u$ in $L^{1}(D)$ and

$$
\lim _{j \rightarrow \infty} \int_{D} \psi_{q}(u(z))\left(d d^{c} g_{j p_{j}}\right)^{n}=\int_{D} \psi_{q}(u(z))\left(d d^{c} u\right)^{n}
$$

for every $q$, our theorem is proved for the functions of the special type.

Suppose that $\phi$ is defined on a neighborhood $V$ of $\bar{D}$. Let $u$ be a continuous plurisubharmonic function on $D$ with zero boundary values. The sequence of plurisubharmonic functions $u_{k}$ equal to $\max \{u, k \phi\}$ on $D$ and $k \phi$ on $V \backslash D$ is decreasing on $D$ and converges to $u$ uniformly on $\bar{D}$. In particular, $\left(d d^{c} u_{k}\right)^{n}$ weak-* converges to $\left(d d^{c} u\right)^{n}$. Hence to prove our theorem for continuous functions it suffices to prove it for continuous functions that admit a continuous plurisubharmonic extension to $V$.

If $u$ is such a function, then there is a decreasing sequence of plurisubharmonic functions $u_{k}$ on some $D^{j}$ that belong to $C^{\infty}\left(D^{j}\right)$ (see [K2, Theorem 2.9.2]) and converge to $u$ uniformly on $\bar{D}$. Adding $\epsilon_{k}|z|^{2}-\delta_{k}$ to $u_{k}$, where the numbers $\epsilon_{k}, \delta_{k}>$ 0 converge to 0 and $\epsilon_{k}|z|^{2}-\delta_{k}<0$ on $D$, we may assume that the functions $u_{k}$ are strictly plurisubharmonic, $u_{k}<0$ on $\bar{D}$, and they still converge uniformly to $u$ on $\bar{D}$.

Let us choose a sequence of numbers $\sigma_{1 k}<0$ converging to 0 such that for all $k$ the set $\left\{u_{k}=\sigma_{1 k}\right\}$ is a smooth hypersurface compactly belonging to $D$. We define $u_{k}^{\prime}$ as a plurisubharmonic function that is equal to $u_{k}$ on the set $W_{k}=\left\{u_{k} \leq \sigma_{1 k}\right\}$, to 0 on $\partial D$, and is maximal on $D \backslash W_{k}$. These functions converge uniformly to $u$. Since for functions like this our theorem is already proved, it is proved for continuous plurisubharmonic functions.

Any plurisubharmonic function $u$ on $D$ belongs to $L_{l o c}^{1}(D)$, and if $u$ has zero boundary values, then it belongs to $L^{1}(D)$. The functions $u_{j}$ that are equal to $\max \{u, j \phi\}, j=1,2, \ldots$, on $D$ and to $j \phi$ outside $D$ form a decreasing sequence of functions on $D$ converging to $u$. Hence they converge to $u$ in $L^{1}(D)$. Using [K2] Theorem 2.9.2] again, we approximate each $u_{j}$ by a decreasing sequence $\left\{u_{k j}\right\}$ of continuous plurisubharmonic functions. Since $u_{j}=0$ on $\partial D$, it is easy to see that the functions $u_{k j}$ can be modified to be equal to 0 on $\partial D$ and still converge to $u_{j}$ in $L^{1}(D)$. The result above implies the existence of Green functions converging to $u_{j}$ and, consequently, to $u$ in $L^{1}(D)$.

\section{REFERENCES}

[ARZ] A. Aytuna, A. Rashkovskii and V. P. Zahariuta, Width asymptotics for a pair of Reinhardt domains, Ann. Polon. Math., 78 (2002), 31-38.

[B] E. Bishop, Mappings of partially analytic spaces, Amer. J. Math., 83 (1961), 209-242. MR 23:A1054

[D] J. P. Demailly, Mesures de Monge-Ampère et mesures plurisousharmoniques, Math. Zeit., 194 (1987), 519-564. MR 88g:32034

[GR] R. C. Gunning and H. Rossi, Analytic Functions of Several Complex Variables, PrenticeHall, Inc., 1974. MR 31:4927 (1st ed.)

[K1] M. Klimek, Extremal plurisubharmonic functions and invariant pseudodistances, Bull. Soc. Math. France, 113 (1985), 123-142.

[K2] M. Klimek, Pluripotential Theory, Oxford Sci. Publ., 1991. MR 93h:32021 
[N] S. Nivoche, Sur une conjecture de Zahariuta et un problème de Kolmogorov, C. R. Acad. Sci. Paris Sér. I Math. 333 (2001), 839-843.

[NP] S. Nivoche and E. A. Poletsky, Multipole Green functions, (preprint)

[S] N. Sibony, Prolongemant de fonctions holomorphes bornees et metrique de Carathéodory, Invent. Math., 29 (1975), 205-230. MR 52:6029

[Z] V. P. Zahariuta, Spaces of analytic functions and maximal plurisubharmonic functions, Doc. Sci. Thesis, 1984.

[Z1] V. P. Zahariuta, Spaces of analytic functions and complex potential theory, Linear Topological Spaces and Complex Analysis, I, (1994), 74-146. MR 96a:46046

[ZS] V. P. Zahariuta and N. P. Skiba, Estimates of $n$-diameters of some classes of functions analytic on Riemann surfaces, Mat. Zametki, 19 (1976), 899-911; English transl., Math. Notes 19 (1976), 525-532. MR 54:7801

Department of Mathematics, 215 Carnegie Hall, Syracuse University, Syracuse, New YORK 13244 